\title{
ition in an Unsteady Mixing Layer Subject to Strain and Variable Pressure
}

\author{
A. LIÑÁN
}

and

\section{F. A. WILLIAMS}

\begin{abstract}
A general formulation is given for ignition in nonpremixed systems involving time-dependent mixing of fuel and oxidizer streams that experience both strain and time-varying pressure, subject to one-step, Arrhenius chemistry. From this general formulation, a number of specific situations are identified that require separate ignition-stage analyses. These ignition-stage analyses are completed for those cases that appear to be of greatest relevance to autoignition in Diesel engines. The resulting ignition times may thus be employed in arriving at estimates of Diesel ignition.
\end{abstract}

\section{NOMENCLATURE}

\section{$A$ strain rate}

$B$ reaction-rate prefactor

$c$ nondimensional temperature difference (Eq. A4)

$c_{p} \quad$ specific heat at constant pressure

$D$ Damköhler number (Eq. A6)

$E$ activation energy

$F$ nondimensional reaction rate defined in Eq. 47

$G$ nondimensional reaction rate defined above Eq. 47

$k$ Chapman-Rubesin parameter defined in Eq. 40

$L \quad$ Lewis number

$m$ reaction order with respect to oxidizer

$n$ reaction order with respect to fuel

$P$ pressure

Pr Prandtl number

$q$ heat release per unit mass of fuel consumed

$R^{o}$ universal gas constant

$S$ entropy-related function defined in Eq. 42

$s$ stoichiometric air-to-fuel mass ratio, Eq. A1

$T$ temperature

$T_{\text {af }}$ adiabatic flame temperature $t$ time

$u$ velocity in $x$ direction

$v$ velocity in $y$ direction

$W$ molecular weight

$w$ reaction rate

$x \quad$ coordinate parallel to mixing layer

$Y$ mass fraction

$y$ coordinate normal to mixing layer

$Z$ mixture fraction

\section{Greek Symbols}

$\alpha$ thermal diffusivity; in Appendix A, nondimensional heat release, Eq. A1

$\beta$ nondimensional activation energy, Eq. A1

$\gamma \quad$ ratio of specific heats

$\Delta \quad$ reduced Damköhler number, Eq. A22

$\delta \quad$ boundary-layer thickness

$\zeta$ nondimensional distance normal to mixing layer defined in Eq. 37

$\eta$ nondimensional distance $y / \delta$ normal to mixing layer

$\theta \quad$ nondimensional entropy variable defined in Eq. 44

$\Lambda$ nondimensional reaction-rate parameter defined in Eq. B3

$\lambda$ thermal conductivity 
$\nu \quad$ stoichiometric coefficient

$\xi \quad$ stretched mixture-fraction variable, Eq. A7

$\rho$ density

$\sigma \quad$ nondimensional time defined in Eq. 37

$\tau$ nondimensional time defined in Eq. 17

$\varphi$ temperature perturbation, Eq. A3

$\phi \quad$ stretched entropy variable defined in Eq. B1

$\chi \quad$ stretched fuel concentration defined in Eq. B13

$\psi \quad$ normalized concentration variable defined in Eq. 44

\section{Subscripts}

$c$ condition at ignition

$I$ ignition

$i$ position at which ignition first occurs

$E$ extinction

$F$ fuel

$m$ maximum temperature

$O$ oxidizer

$o$ boundary value

$r$ reaction zone

$s \quad$ stagnation point

0 initial value

\section{INTRODUCTION}

Diesel combustion involves transient injection of a liquid fuel stream at high pressure into a chamber containing oxidizing gas in turbulent motion, with the chamber pressure increasing as a consequence of the volume decrease produced by piston motion [1]. Computations of such processes show regions of variable strain rates where vaporized fuel and oxidizer mix, such as at the front of the injected fuel jet [2]. To predict when ignition will occur in processes of this kind, it is helpful to have results of analyses of ignition in unsteady, strained, variable-pressure mixing. The intent of the present paper is to offer such results on the basis of activation-energy asymptotics for onestep processes [3]. Analyses of this kind can be useful in that they provide parametric descriptions of ignition times, not tied to specific values of transport and reaction-rate parameters. In applications, the results are to be used in conjunction with spray and chemistry descriptions, not addressed here, that provide values for the parameters that appear in the analysis.

The nonpremixed combustion to be considered here results in diffusion flames of the type analyzed previously [4] for steady, strained, counterflow configurations involving reactants with Lewis numbers of unity. That analysis [4] identified four regimes, one of which, the ignition regime, is relevant to the problems addressed here. Because of the low diffusion coefficients of fuels typically encountered in Diesel combustion, there is interest in analyzing mixtures with Lewis numbers different from unity. Although Lewis-number effects in such diffusion flames have been considered previously [5-7] these studies did not treat the ignition regime. The present investigation is focused exclusively on the ignition regime and admits nonunity Lewis numbers.

Unlike the analyses cited above, the present study also admits time-dependent behavior. Time-dependent reactant consumption with strain has been treated earlier [8] for Lewis numbers of unity in the diffusion-controlled limit of large Damköhler numbers, where ignition events do not occur. Also, ignition in time-dependent, unstrained mixing layers has been analyzed previously [9] by activationenergy asymptotics for reactants with Lewis numbers of unity. The present work may be considered to generalize these last results [9] to different Lewis numbers and to timedependent problems with strain. In addition to working out the solutions for the cases that appear to be of the greatest practical interest, the present paper will indicate the additional cases that may arise and the asymptotic methods for solving those problems.

Niioka [10] took the first step towards generalizing the previous [9] analysis to include strain. Although he restricted his attention to Lewis numbers and reaction orders of unity and to a particular initial-value problem, his analysis by activation-energy asymptotics clearly showed the delay in ignition that is caused by the imposition of strain. The present work extends that of Niioka to different Lewis numbers and reaction orders and to more gen- 
eral initial and boundary conditions, as well as showing how compression can modify and simplify the ignition-stage analysis.

The general formulation is given in the following section, and analyses for various cases are addressed in subsequent sections.

\section{FORMULATION}

Attention is directed to the ignition of two streams, one of fuel and the other of oxidizer. These two streams flow opposite to each other and form a mixing layer that will be analyzed in a reference system that moves with the dividing fluid surface. Consideration is restricted to the stagnation region, where the flow and concentration field can be described in terms of a similarity solution involving only the variable time $t$ and the transverse coordinate $y$. The temperatures of the fuel and oxidizer streams, $T_{F}$ and $T_{O}$, will be considered to be different and changing with time because of the compression work associated with the variable stagnation-point pressure $P_{s}(t)$, all time scales being assumed long compared with acoustic times. If $x$ is the coordinate along a locally planar mixing layer measured from the stagnation point and $u$ the corresponding velocity component, which is associated with the strain, then this velocity takes the values $u=A_{O} x$ and $u=A_{F} x$ in the air and fuel streams, respectively, related to variations $P^{\prime}$ of pressure from its stagnation-point value according to

$$
\begin{aligned}
-\frac{\partial P^{\prime}}{\partial x} & =\rho_{O}\left(\frac{d A_{O}}{d t}+A_{O}^{2}\right) x \\
& =\rho_{F}\left(\frac{d A_{F}}{d t}+A_{F}^{2}\right) x,
\end{aligned}
$$

where $\rho_{F}$ and $\rho_{O}$ are the densities of the fuel and oxidizer streams. Equation 1 relates the apparently different strain rates of the two streams, since there are no $x$-dependent pressure changes across the mixing layer. Inside the mixing layer $u=x A(y, t)$, where $A$ must satisfy boundary conditions consistent with the second equality in Eq. 1.

The conservation equation for the density $\rho$, the variable strain rate $A$, the temperature $T$, the mixture fraction $Z$ and the mass fractions of fuel $Y_{F}$ and of oxidizer $Y_{O}$ are, respectively,

$$
\begin{aligned}
& \frac{\partial \rho}{\partial t}+\frac{\partial \rho v}{\partial y}+\rho A=0, \\
& \rho\left(\frac{\partial A}{\partial t}+v \frac{\partial A}{\partial y}+A^{2}\right) \\
& =\rho_{O}\left(\frac{d A_{O}}{d t}+A_{O}^{2}\right) \\
& +\operatorname{Pr} \frac{\partial}{\partial y}\left(\frac{\lambda}{c_{p}} \frac{\partial A}{\partial y}\right) \\
& \rho\left(\frac{\partial T}{\partial t}+v \frac{\partial T}{\partial y}\right)=\frac{\partial}{\partial y}\left(\frac{\lambda}{c_{p}} \frac{\partial T}{\partial y}\right) \\
& +\frac{1}{c_{p}} \frac{d P_{s}}{d t}-\frac{q}{c_{p}} w_{F}, \\
& \rho\left(\frac{\partial Z}{\partial t}+v \frac{\partial Z}{\partial y}\right)=\frac{\partial}{\partial y}\left(\frac{\lambda}{c_{p}} \frac{\partial Z}{\partial y}\right), \\
& \rho\left(\frac{\partial Y_{F}}{\partial t}+v \frac{\partial Y_{F}}{\partial y}\right)=\frac{1}{L_{F}} \frac{\partial}{\partial y}\left(\frac{\lambda}{c_{p}} \frac{\partial Y_{F}}{\partial y}\right)+w_{F}, \\
& \rho\left(\frac{\partial Y_{O}}{\partial t}+v \frac{\partial Y_{O}}{\partial y}\right)=\frac{1}{L_{O}} \frac{\partial}{\partial y}\left(\frac{\lambda}{c_{p}} \frac{\partial Y_{O}}{\partial y}\right) \\
& +\nu_{O} w_{F} \text {, }
\end{aligned}
$$

where Pr denotes the Prandtl number and $L_{k}$ the Lewis number for species $k$, all assumed constant, $\lambda$ represents the (variable) thermal conductivity, and $c_{p}=\sum_{k=1}^{N} c_{p k} Y_{k}$ the specific heat at constant pressure, taken here to be constant, an approximation that may be reasonable because of the relatively small temperature changes that typically occur prior to thermal runaway in the ignition process. In these equations, the mass rate of consumption of fuel is denoted by $w_{F}$, the mass of oxidizer consumed per unit mass of fuel is represented by the stoichiometric coefficient $\nu_{O}$, and the heat released per unit mass of fuel consumed is identified by $q$. Equations 2-4 express conservation of mass, the $x$ component of momen- 
tum and energy, respectively; the $y$ component of momentum conservation serves only to determine the small pressure changes in the direction normal to the mixing layer, and therefore it need not be considered. With Lewis numbers differing from unity there does not exist a unique mixture fraction, but it is convenient to employ the thermal diffusivity in selecting a mixture-fraction definition, as is done in Eq. 5. The equation of state for an ideal gas is employed,

$P_{s} / \rho T=R^{o} / \bar{W}$,

under the assumption that the average molecular weight $\bar{W}=\left(\sum_{k=1}^{N} Y_{k} / W_{k}\right)^{-1}$ is constant; $R^{o}$ denotes the universal gas constant. The reaction rate is assumed of the Arrhenius form

$w_{F}=-\rho B Y_{F}^{n} Y_{O}^{m} \exp \left(-E / R^{o} T\right)$,

where $m$ and $n$ are reaction orders, $E$ the overall activation energy, and $B$ a pressuredependent prefactor having units of reciprocal time.

The variable pressure $P_{s}(t)$ (increasing with time) will be considered to be known, as will the variable strain rate $A_{O}(t)$. The boundary conditions are

$$
\begin{array}{rlrl}
T & \rightarrow T_{O}(t), \quad Z \rightarrow 0, & Y_{F} \rightarrow 0, \\
Y_{O} \rightarrow Y_{O o} \text { and }
\end{array}
$$

and

$$
\begin{array}{rrr}
T \rightarrow T_{F}(t), \quad Z \rightarrow 1, & Y_{F} \rightarrow Y_{F o}, \\
Y_{O} \rightarrow 0 \quad \text { and }
\end{array}
$$

with $A_{F}, T_{F}$, and $\rho_{F}$ related to $A_{O}, T_{O}$, and $\rho_{O}$ by

$$
\begin{aligned}
\rho_{F} T_{F} & =\rho_{O} T_{O}=P_{s} \bar{W} / R^{o}, \\
\rho_{F}\left(\frac{d A_{F}}{d t}+A_{F}^{2}\right) & =\rho_{O}\left(\frac{d A_{O}}{d t}+A_{O}^{2}\right),
\end{aligned}
$$

and

$$
\rho_{F} c_{p} \frac{d T_{F}}{d t}=\rho_{O} c_{p} \frac{d T_{O}}{d t}=\frac{d P_{s}}{d t} .
$$

The origin of the $y$ coordinate system is chosen so that

$v=0$ at $y=0$.

In addition to these boundary conditions, initial conditions are needed to define a wellposed problem. Typical initial conditions are those associated with a sharp interface between the fuel and the air at time $t=0$,

$$
\begin{aligned}
T & =T_{O}(0), \quad Z=0, \quad Y_{F}=0, \\
Y_{O} & =Y_{O o}, \quad \text { and } \quad A=A_{O}(0) \quad \text { for } y>0,
\end{aligned}
$$

and

$$
\begin{array}{cl}
T=T_{F}(0), & Z=1, \quad Y_{F}=Y_{F o}, \\
Y_{O} & =0, \quad \text { and } \quad A=A_{F}(0) \quad \text { for } y<0 .
\end{array}
$$

This completes the set of equations that in principle determine $v, T, Z, Y_{F}, Y_{O}$, and $\rho$ as functions of space and time.

\section{LENGTH AND TIME SCALES}

Since ignition often occurs near the hightemperature oxidizer stream, it is convenient to employ properties of this stream in defining nondimensional length and time variables. A time-dependent boundary-layer thickness $\delta(t)$ may be taken to obey the ordinary differential equation

$$
\delta \frac{d \delta}{d t}+\delta^{2}\left(A_{O}+\frac{d \ln \rho_{O}}{d t}\right)=\alpha_{O},
$$

where the thermal diffusivity of the oxidizer stream is

$\alpha_{O}=\lambda_{O} /\left(\rho_{O} c_{p}\right)$.

An appropriate nondimensional length variable is then

$\eta=y / \delta$.

A corresponding time variable is

$\tau=\int_{0}^{t}\left(\alpha_{O} / \delta^{2}\right) d t$ 
It is straightforward to transform Eqs. 2-7 from $(y, t)$ to $(\eta, \tau)$ as independent variables. That the resulting system will be useful for most of the ignition problems of interest here can be seen from the following simplified development of the mixture-fraction equation for inert mixing fields.

Consider the case of nearly equal temperatures and molecular weights of both streams. In this case during the ignition transients the change in temperatures produced by the chemical reaction is still small, and then Eq. 3 has the solution $A=A_{O}(t)=A_{F}(t)$, constant in $y$, and Eq. 2 simplifies to

$v=-\left(A_{O}+\frac{d \ln \rho_{O}}{d t}\right) y$

because in the ignition transient we can neglect the spatial variations in $\rho$, as well as those of T. Equation 5 then reduces to

$$
\frac{\partial Z}{\partial t}-\left(A_{O}+\frac{d \ln \rho_{O}}{d t}\right) y \frac{\partial Z}{\partial y}=\alpha_{O} \frac{\partial^{2} Z}{\partial y^{2}},
$$

which upon introduction of Eq. 16 becomes

$$
\begin{aligned}
\left(\delta^{2} / \alpha_{O}\right) & Z_{t} \\
& -\eta\left\{\left(\delta^{2} / \alpha_{O}\right)\right. \\
& \left.\times\left(\frac{\delta_{t}}{\delta}+A_{O}+\frac{d \ln \rho_{O}}{d t}\right)\right\} Z_{\eta} \\
= & Z_{\eta \eta},
\end{aligned}
$$

where the subscripts $t$ and $\eta$ identify partial derivatives. Equation 20 clearly motivates the selection given in Eq. 14. With this formula and Eq. 17, the further transformation from $t$ to $\tau$ reduces Eq. 20 to the simple form

$$
Z_{\tau}-\eta Z_{\eta}=Z_{\eta \eta}
$$

corresponding to a mixing layer under a constant strain of unity. Because the boundary conditions for Eq. 21 are constant, $Z \rightarrow 1$ as $\eta \rightarrow-\infty$ and $Z \rightarrow 0$ as $\eta \rightarrow \infty$, the solution soon becomes $\tau$-independent and is then given by

$Z=\frac{1}{2} \operatorname{erfc}(\eta / \sqrt{2})$

Similar treatments of Eqs. 6 and 7 for frozen flow in the cases $L_{F} \neq L_{O} \neq 1$ readily yield

$Y_{F} / Y_{F o}=\frac{1}{2} \operatorname{erfc}\left(\eta \sqrt{L_{F} / 2}\right) \equiv Z_{F}$

and

$1-Y_{O} / Y_{O o}=\frac{1}{2} \operatorname{erfc}\left(\eta \sqrt{L_{O} / 2}\right) \equiv Z_{O}$

These results show how Eq. 14 defines the evolution of the boundary-layer thickness.

Equation 14 may be integrated formally to show that $\delta^{2}$ may be expressed in the form

$\delta^{2}=C \rho_{O}^{-2} \exp \left\{-2 \int_{0}^{t} A_{O} d t\right\}$

where

$\frac{d C}{d t}=2 \alpha_{O} \rho_{O}^{2} \exp \left\{2 \int_{0}^{t} A_{O} d t\right\}$,

and $C=C_{0}=\delta_{0}^{2} \rho_{O}^{2}(0)$ at $t=0$, where $\delta_{0}$ is a characteristic value of $\delta$ at that time. Integration of Eq. 25 gives

$$
\begin{aligned}
C= & C_{0}+2 \int_{0}^{t} \alpha_{O}\left(t^{\prime}\right) \rho_{O}^{2}\left(t^{\prime}\right) \\
& \times \exp \left\{2 \int_{0}^{t^{\prime}} A_{O}\left(t^{\prime \prime}\right) d t^{\prime \prime}\right\} d t^{\prime},
\end{aligned}
$$

which may be used in Eq. 24 to provide an explicit expression for $\delta(t)$ that involves integrals. These results allow for arbitrary time variations of strain through $A_{O}$ and of pressure through $\rho_{O}$, and $\alpha_{O}$. Often the characteristic times for pressure variations are long compared with $A_{O}^{-1}$ and with the characteristic time for $A_{O}$ variation, so that in these results $\alpha_{O}$ becomes constant and $\rho_{O}$ disappears, and the term in Eq. 4 involving the time derivative of the pressure then also becomes negligible; in this case, when solving Eqs. 2-7, $P_{s}, T_{O}$, and $\rho_{O}$ are parameters, not functions of the short time scale relevant to the description of the transient mixing layer. 


\section{SIMPLIFICATIONS FOR CONSTANT RATE OF STRAIN}

An important limiting case is that of constant rate of strain, $A_{O}$ constant, or strain changing over times large compared with $\boldsymbol{A}_{O}^{-1}$. For this case, Eqs. 24 and 26 yield simply $\delta^{2}=\alpha_{O} /$ $A_{O}=$ constant. If the strain time $A_{O}^{-1}$ is also small compared with the characteristic compression time, then the term $c_{p}^{-1} \partial P_{s} / \partial t$ can be left out of Eq. 4, together with the remaining time derivative terms in Eqs. 2-7, so that the system becomes expressible as

$$
\begin{aligned}
\frac{d \rho v}{d y}+\rho A & =0 \\
\rho\left(v \frac{d A}{d y}+A^{2}\right) & =\rho_{O} A_{O}^{2}+\operatorname{Pr} \frac{d}{d y}\left(\frac{\lambda}{c_{p}} \frac{d A}{d y}\right) \\
\rho v \frac{d Y_{F}}{d y} & =\frac{1}{L_{F}} \frac{d}{d y}\left(\frac{\lambda}{c_{p}} \frac{d Y_{F}}{d y}\right)+w_{F} \\
\rho v \frac{d Y_{O}}{d y} & =\frac{1}{L_{O}} \frac{d}{d y}\left(\frac{\lambda}{c_{p}} \frac{d Y_{O}}{d y}\right)+\nu_{O} w_{F}, \\
\rho v \frac{d T}{d y} & =\frac{d}{d y}\left(\frac{\lambda}{c_{p}} \frac{d T}{d y}\right)-\frac{q}{c_{p}} w_{F},
\end{aligned}
$$

and

$$
\begin{aligned}
& \rho T=P_{s} \bar{W} / R^{o}, \\
& w_{F}=-\rho B Y_{F}^{n} Y_{O}^{m} e^{-E / R^{o} T},
\end{aligned}
$$

a system of ordinary differential equations (those describing the steady stagnation-point boundary-layer flow) to be solved with the boundary conditions of Eq. 10, where now $A_{O}$ and $A_{F}$ are taken as constant and related by

$\rho_{F} A_{F}^{2}=\rho_{O} A_{O}^{2}$,

while $T_{F}$ and $T_{O}$ are related to $P_{s}$ by Eqs. 11a and $11 \mathrm{c}$, namely,

$T_{O} / T_{O 0}=\left(P_{s} / P_{s 0}\right)^{(\gamma-1) / \gamma}=T_{F} / T_{F 0}$,

while

$A_{F}^{2} / A_{O}^{2}=\rho_{O} / \rho_{F}=\rho_{O 0} / \rho_{F 0}$.
The system of equations given by Eqs. 27-32, with the boundary conditions of Eqs. 10, 33, and 34, will have, for large activation energies, a nearly frozen solution for values of the pressure smaller than a critical value, $P_{I}$, such that when $P_{s}=P_{I}$ is reached during the compression process, spontaneous ignition will take place in the mixing layer. There is a second critical pressure, $P_{E}<P_{I}$, such that if $P_{s}>P_{E}$ the above system of equation has fast-burning diffusion-controlled solutions, for which the chemical reaction is confined to a thin reaction sheet. In the pressure interval $P_{E}<P_{s}<P_{I}$ the problem of Eqs. 27-32 and 10 has three solutions the nearly frozen one, the nearequilibrium, diffusion-controlled solution, and a third intermediate unstable solution. These changes with pressure arise mainly through Eq. 33 and the strong dependence of the reaction rate on temperature in Eq. 32; direct pressure dependences, as through $B$, are much less important. Because of the quasisteady character of the problem, the critical pressures $P_{E}$ and $P_{I}$ are expressed most conveniently in terms of corresponding critical Damköhler numbers. Analyses that determine critical Damköhler numbers for ignition, thereby giving $P_{I}$, deserve further discussion here.

For the special case of unity Lewis numbers and unity reaction orders, the relevant ignition analysis has been published previously [4], in the so-called thermal-diffusion approximation in which for simplicity the density and transport coefficients are treated as being constants. Although inaccurate after flame development, this approximation is not too bad for ignition because the fractional temperature increases are small, of order $R^{o} T / E$. That earlier study demonstrated the existence of two ignition regimes, one for which $\left(T_{O o}-T_{F o}\right)$ is of the order of $R^{0} T_{O o}^{2} / E$ or smaller, and the other for which $\left(T_{O o}-T_{F o}\right) \gg R^{o} T_{O o}^{2} / E$. In the former regime, significant heat release occurs throughout the entire mixing layer during ignition, and reactant consumption is negligible prior to ignition, while in the latter regime heat release is significant only at the oxidizer edge of the mixing layer where the temperature and rates are highest, and fuel depletion may be important during ignition because the fuel concentration is small in the ignition 
region. This same classification of ignition regimes applies under the more general conditions addressed here, and it applies for timedependent mixing as well [9].

For the ignition regime in which $\left(T_{O o}-T_{F o}\right)$ is of the order of $R^{o} T_{O o}^{2} / E$ or smaller, use of activation-energy asymptotics results in a two-point boundary-value problem for a second-order ordinary differential equation, the numerical integration of which gives the maximum temperature as a function of a reduced Damköhler number, showing no solutions above a critical Damköhler number for ignition and two solutions below, that of the higher temperature being unstable. These solutions and ignition Damköhler numbers have been calculated and plotted for unity reaction orders with Lewis numbers of unity [4]. Although it would be straightforward to perform such calculations for reaction orders and Lewis numbers different from unity, no such results are currently available. Results for these extended parameter ranges will not be calculated herein either, because this regime typically is not very relevant to Diesel ignition.

However, results for the extended parameter ranges are presented here for the ignition regime in which $\left(T_{O o}-T_{F o}\right) \gg R^{o} T_{O o}^{2} / E$. Not only is this inequality usually satisfied for Diesel autoignitions, but also $L_{F}$ usually is sufficiently large that the previous [4] approximations may be thought to become inaccurate. Results for this regime have been derived earlier $[4,9]$ for reaction orders and Lewis numbers of unity, taking the effect of fuel depletion during the ignition process fully into account. The results to be given here do not allow for fuel depletion, and it could be of interest to extend the analysis to include that effect. The analysis is given in Appendix A, where the condition necessary for fuel depletion to be neglected is derived, and where the equations needed for developing the more general theory are written. In the meantime, use of Fig. 16 and Eq. A11 of the previous paper [4] along with the present results provide an indication of the effects of both fuel depletion and Lewis numbers.

The results of the analysis in Appendix A are plotted in Figs. 1 and 2. Figure 1 determines the nondimensional increment of the

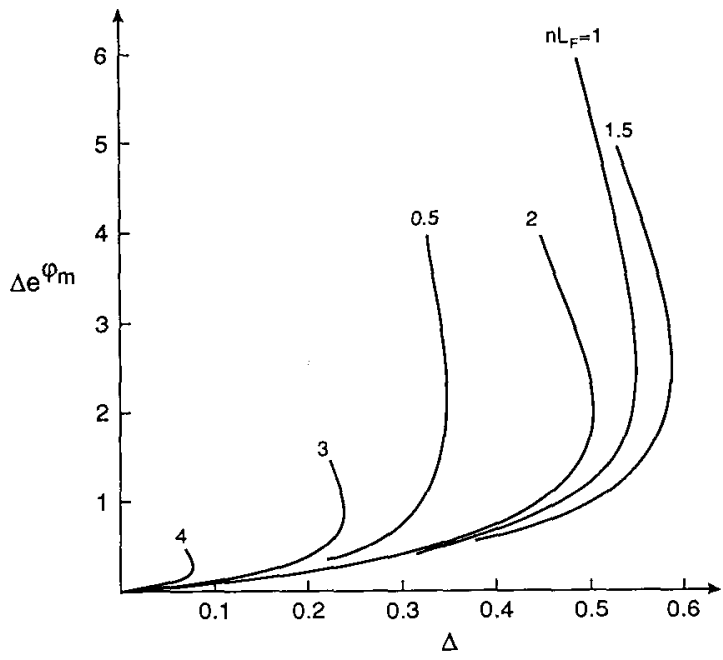

Fig. 1. Results of numerical integration for ignition temperature increments as functions of the relevant Damköhler number, for various values of the reaction order and Lewis number of the fuel, with constant rate of strain.

maximum temperature above that for frozen mixing as a function of the reduced Damköhler number defined in Eq. A22, for various values of the relevant parameter $n L_{F}$. This nondimensional increment is given to leading order

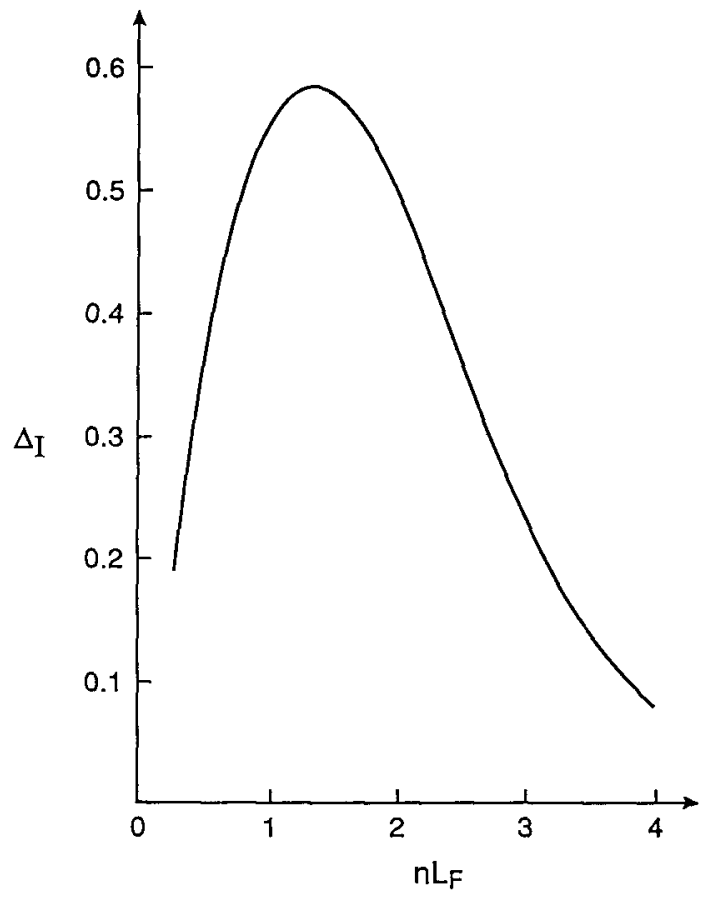

Fig. 2. Ignition Damköhler numbers as functions of the product of the fuel reaction order and Lewis number, for constant rate of strain. 
as

$\varphi_{m}=R^{o}\left(T_{m}-T_{O}\right) / E$,

where $T_{m}$ is the maximum temperature. The lower branches of the curves in Fig. 1 are the stable solutions, and the turning points give the maximum values of $\Delta$, the Damköhler numbers $\Delta$, for ignition. If reactant depletion had been included, and if the heat release were small enough that it could contribute a maximum temperature increment no greater than a critical value dependent on the parameters of the problem, then $\varphi_{m}$ would increase monotonically with $\Delta$ instead of exhibiting the abrupt ignition behavior of Fig. 1. In practice, the heat release generally is larger than this, and the behavior seen in Fig. 1 is representative.

The ignition Damköhler numbers are shown as a function of $n L_{F}$ in Fig. 2. The result for $n L_{F}=1$ has been obtained previously [4] and has $\Delta_{I}=0.536$. It is interesting to observe that $\Delta_{I}=0.5$ for both $n L_{F}=0.8$ and $n L_{F}=2$. This indicates that for a wide range of values of $n L_{F}$ of practical importance, $\Delta_{I}=0.5$ is a good approximation. This value is obtained entirely analytically for $n L_{F}=2$ in Appendix A.

\section{SIMPLIFICATIONS FOR UNSTRAINED MIXING LAYERS WITH VARIABLE PRESSURE}

For unstrained mixing layers, $A_{O}=0$, and Eqs. 24 and 26 show that

$$
\left(\rho_{O} \delta\right)^{2}=\left[\rho_{O}(0) \delta_{0}\right]^{2}+2 \int_{0}^{t} \alpha_{O}\left(t^{\prime}\right) \rho_{O}^{2}\left(t^{\prime}\right) d t^{\prime} .
$$

If $\alpha_{O} \rho_{O}^{2}$ is constant, then Eq. 36 implies that $\left(\rho_{O} \delta\right)^{2}$ increases linearly with time, as in a diffusion process. Although it is possible to work with the $\eta$ and $\tau$ of Eqs. 16 and 17 as independent variables, greater simplification is achieved (especially in the absence of strain) by introducing a mass coordinate for the unsteady mixing layer. Therefore, a characteristic time $t_{0}$ is selected, to be defined later, and the nondimensional variables

$$
\sigma=t / t_{0}, \quad \zeta=\left(t_{0} \alpha_{O 0} \rho_{O 0}^{2}\right)^{-1 / 2} \int_{0}^{y} \rho d y
$$

are introduced, where the subscripts 0 on $\alpha_{O}$ and $\rho_{O}$ mean that they are to be evaluated at $t=0$. In terms of $\sigma$ and $\zeta$, Eqs. 4, 6, and 7 formally become

$$
\begin{aligned}
\frac{\partial T}{\partial \sigma}-\frac{\partial}{\partial \zeta}\left(k \frac{\partial T}{\partial \zeta}\right)= & \frac{\gamma-1}{\gamma} \frac{T}{P_{s}} \frac{d P_{s}}{d \sigma} \\
& -\frac{q}{c_{p}} t_{0} \frac{w_{F}}{\rho}
\end{aligned}
$$

and

$$
\begin{aligned}
\frac{\partial Y_{F}}{\partial \sigma}-\frac{1}{L_{F}} \frac{\partial}{\partial \zeta}\left(k \frac{\partial Y_{F}}{\partial \zeta}\right) & \\
= & \frac{\partial\left(Y_{O} / \nu_{O}\right)}{\partial \sigma} \\
& -\frac{1}{L_{O}} \frac{\partial}{\partial \zeta}\left[k \frac{\partial\left(Y_{O} / \nu_{O}\right)}{\partial \zeta}\right] \\
& =t_{0} \frac{w_{F}}{\rho}
\end{aligned}
$$

where a Chapman-Rubesin type of parameter is

$k=\left(\lambda \rho / c_{p}\right) /\left(\alpha_{O 0} \rho_{O 0}^{2}\right)$,

and the ratio of specific heats, $\gamma$, obeys

$$
(\gamma-1) / \gamma=R^{o} /\left(\bar{W} c_{p}\right)
$$

use having been made of Eq. 8. Equations 10 and 13 readily provide boundary and initial conditions for Eqs. 38 and 39.

A detailed treatment of the resulting ignition problem has been given earlier [9] for the special case $k=L_{F}=L_{O}=1, d P_{s} / d \sigma=0$. It is interesting to note [11] that pressure variations are readily taken into account in the analysis by working with the entropy-related function

$S=T / P_{s}^{(\gamma-1) / \gamma}$

instead of $T$. Although in ideal gas mixtures $k$ is proportional to $P_{s}$ at constant $T$ and composition, this pressure effect on the transport coefficients often is small enough to be neglected, and the approximation $k=1$ may 
be introduced for simplicity. With this approximation, use of Eq. 42 in Eq. 38 results in

$$
\frac{\partial S}{\partial \sigma}-\frac{\partial^{2} S}{\partial \zeta^{2}}=-\frac{q t_{0} w_{F}}{c_{p} \rho P_{s}^{(\gamma-1) / \gamma}},
$$

to be solved along with Eq. 39 for $k=1$. If $\rho \lambda / c_{p}$ is constant at constant pressure (a fairly reasonable approximation for ideal gases), then $k$ is a function only of $P_{s}$, which in turn is a function only of $\sigma$, so the factor $k(\sigma)$ may be taken outside the derivative in Eqs. 38 and 39, and introduction of the revised time variable $\int_{0}^{\sigma} k d \sigma$ automatically takes into account the time variations of the diffusivities and results in a problem having the same functional form as that for $k=1$. With this simple generalization in mind, equations are written here only for $k=1$. It may be noted that the mass and time coordinates introduced here are also useful [12] for $A_{O} \neq 0$, even though, for simplicity, they were not introduced in the preceding development.

The increase of $P_{s}$ with increasing $\sigma$ can enhance ignition by increasing temperatures, as seen in Eq. 11c. If this is an important effect, then it is convenient to select $t_{0}$ to be the characteristic time over which fractional pressure change of order unity occur, in particular making $[(\gamma-1) / \gamma] d\left(\ln P_{s}\right) / d \sigma$ of order unity in Eq. 38. If the pressure changes are slow enough to constitute small perturbations, then it is more convenient to select $t_{0}$ to be a relevant chemical time, as was done previously [9]. In any case, normalized nondimensional variables can be introduced as

$$
\theta=S / S_{O}, \quad \psi_{F}=Y_{F} / Y_{F o}, \quad \psi_{O}=Y_{O} / Y_{O o},
$$

and the partial differential equations

$$
\begin{aligned}
\frac{\partial \theta}{\partial \sigma}-\frac{\partial^{2} \theta}{\partial \zeta^{2}}= & F, \\
\frac{\partial \psi_{F}}{\partial \sigma}-\frac{1}{L_{F}} \frac{\partial^{2} \psi_{F}}{\partial \zeta^{2}}= & \frac{\partial\left(\psi_{O} / s\right)}{\partial \sigma} \\
& -\frac{1}{L_{O}} \frac{\partial^{2}\left(\psi_{O} / s\right)}{\partial \zeta^{2}} \\
= & -G
\end{aligned}
$$

derived, subject to boundary conditions

$$
\begin{array}{lll}
\psi_{F} \rightarrow 0, & \psi_{O} \rightarrow 1, & \theta \rightarrow 1 \text { as } \zeta \rightarrow \infty ; \\
\psi_{F} \rightarrow 1, & \psi_{O} \rightarrow 0, & \theta \rightarrow \theta_{F} \text { as } \\
& & \zeta \rightarrow-\infty
\end{array}
$$

and to similar initial conditions, as obtained from Eqs. 10 and 13. Here $s=\nu_{O} Y_{F_{o}} / Y_{O_{g}}$, $G=\left(P_{s} / P_{s 0}\right)^{(\gamma-1) / \gamma}\left[\left(c_{p} T_{O 0}\right) /\left(q Y_{F o}\right)\right] F$, and, from Eq. 9,

$$
\begin{aligned}
F= & \left(\frac{P_{s}}{P_{s_{0}}}\right)^{-(\gamma-1) / \gamma} \frac{q B Y_{F_{o}}^{n} Y_{O_{o}}^{m} t_{0}}{c_{p} T_{O_{0}}} \psi_{F}^{n} \psi_{o}^{m} \exp \\
& \times\left[-\left(\frac{E}{R^{o} T_{O_{0}}}\right)\left(\frac{P_{s}}{P_{s_{0}}}\right)^{-(\gamma-1) / \gamma}\left(\frac{1}{\theta}\right)\right] .
\end{aligned}
$$

Equation 11 is employed in deriving the boundary conditions for $\theta$, and $\theta_{F} \equiv T_{F} / T_{O}$, which is constant by virtue of Eqs. 11a and 11c, the adiabaticity condition in the external streams. The problem defined by Eqs. 45 and 46 offers a most convenient way to address the unstrained ignition and exhibits the relevant nondimensional parameters, $\theta_{F}, s, L_{F}, L_{O}$, and the parameters appearing in $F$ and $G$.

When ignition is produced by compression, there is an inert initial stage during which $F$ and $G$ are negligibly small. The solutions to Eqs. 45 and 46 during this inert stage are

$$
\begin{aligned}
\theta & =1-\left(1-\theta_{F}\right) \frac{1}{2} \operatorname{erfc}\left(\frac{\zeta / 2}{\sqrt{\sigma}}\right), \\
\psi_{F} & =\frac{1}{2} \operatorname{erfc}\left(\frac{\zeta}{2} \sqrt{\frac{L_{F}}{\sigma}}\right), \\
\psi_{0} & =1-\frac{1}{2} \operatorname{erfc}\left(\frac{\zeta}{2} \sqrt{\frac{L_{O}}{\sigma}}\right) .
\end{aligned}
$$

Within the context of activation-energy asymptotics, this inert stage ends at a time $\sigma_{c}$, and the large parameter of expansion, $\beta$, is defined by

$$
\begin{aligned}
\beta & =E /\left(R^{o} T_{O c}\right), \\
T_{O c} & \equiv T_{O 0}\left[P_{s}\left(\sigma_{c}\right) / P_{s 0}\right]^{(\gamma-1) / \gamma} .
\end{aligned}
$$

A first approximation to the compressionproduced ignition time $\sigma_{c}$ may be obtained by substituting Eqs. 48 and 49 into Eq. 47 then 
putting $F=F_{c}$, where

$F_{c} \equiv[(\gamma-1) / \gamma]\left[d\left(\ln P_{s}\right) / d \sigma\right]_{\sigma=\sigma_{c}}$,

which is the order of magnitude of $F$ when the chemistry begins to become important. A more thorough analysis involves application of activation-energy asymptotics in a short ignition stage near $\sigma=\sigma_{c}$, as has been done for $L_{F}=$ $L_{O}=1$ in an earlier report [11]. Again, there are two regimes, one in which $\beta\left(1-\theta_{F}\right)$ is of order unity or smaller, and the other in which $\beta\left(1-\theta_{F}\right)$ is large. In the first of these, the heat release in the ignition stage occurs throughout the mixing layer (with negligible reactant depletion), and in the second it is localized at the oxidizer edge of the layer (with fuel depletion possibly important). The character of the analysis resembles that of Appendix $A$, although the details are quite different.

An equation for the departure of $\theta$ from the inert solution given in Eq. 48 is readily derived from the first expression in Eq. 45 by suitably expanding $F$ of Eq. 47 for the ignition stage. When $\beta\left(1-\theta_{F}\right)$ is of order unity or smaller and compression is not dominant in producing ignition, the resulting partial differential equation must be solved numerically; such solutions have been plotted previously [9] for $L_{F}=$ $L_{O}=1$ and $m=n=1$. When $\beta\left(1-\theta_{F}\right)$ is large, there are simplifications that circumvent numerical solutions of partial differential equations [9]. When the ignition is produced by compression, the relevant stretched nondimensional time variable of order unity in the ignition state is [11] $F_{C} \beta\left(\sigma-\sigma_{c}\right)$, and diffusive effects during the ignition stage are small, of order $\beta^{-1}$, so that ordinary differential equations in the stretched time variable are obtained [11]. These equations have solutions that can exhibit thermal runaway at a particular time, identified with ignition, as shown in Appendix B, where the details of the procedures differ in the two different ignition regimes.

\section{SIMPLIFICATIONS FOR COMPRESSION- PRODUCED AUTOIGNITION WITH VARIABLE STRAIN}

Thus far, steady-state problem with constant rates of strain and time-dependent problems without strain have been addressed. The gen- eral class of problems defined by Eqs. 2-13 can exhibit a wide variety of different ignition behaviors. Consider first situations in which effects of compression are negligible. The results of Appendix A show that when strain rates are too high, ignition does not occur. As strain rates are relaxed, a condition is reached at which ignition can occur, and it then becomes relevant to ask about ignition times. For values of $\Delta$ of Fig. 1 slightly above the critical value $\Delta_{I}$ of Fig. 2, ignition occurs, but it may require a long time because it is delayed by effects of strain. Under such conditions multiple-stage analyses, analogous to those needed for ignition in homogeneous systems near critical conditions [13], would be appropriate for calculating ignition times. Problems of this kind would be soluble but challenging. At sufficiently large values of $\Delta$, in the first approximation strain can be neglected in calculating ignition times, and the procedures discussed in the preceding section can be applied. It would be straightforward to add small strain rates as perturbations to these methods, to investigate the delay in the ignition time produced by small strain, but this has not been done. Only a few results for mixing-layer ignition times are available at constant pressure without strain [9].

Pressure variations may be expected to complicate the problem even more. For small rates of pressure variation, their effects may be included as perturbations in constant-pressure analyses. Large rates of pressure variation cannot, but the analysis of Appendix B suggests that sufficiently rapid compression can simplify ignition-stage analyses by converting the problems to ordinary differential equations in time. Although Appendix B concerns only strain-free ignition, it may be anticipated that a similar approach will apply in the presence of strain, so long as the compression effects are strong enough and the rate of strain is not so large as to prevent ignition or to delay it excessively. It will be of interest to indicate here how variable strain can be included in such analyses of compression-produced ignition.

For illustrative purposes, the approximations leading to Eq. 18 are retained, and reactant depletion is neglected, so that Eq. 23 applies after the transient mixing development. 
Introduction of the independent variables in Eqs. 16 and 17 and the dependent variables of Eq. 44 then reduce Eq. 4 to

$$
\frac{\partial \theta}{\partial \tau}-\eta \frac{\partial \theta}{\partial \eta}-\frac{\partial^{2} \theta}{\partial \eta^{2}}=F,
$$

where $F$ is given by Eq. 47 with $t_{0}$ put equal to $\delta^{2} / \alpha_{O}$. Boundary and initial conditions are as in Eq. 46.

For compression-produced autoignition, there is an inert initial stage during which $F$ is negligible in Eq. 52. During this stage, $\theta$ will vary from the solution given in Eq. 48 (with $\zeta$ and $\sigma$ replaced by $\eta$ and $\tau)$ to $1-\left(1-\theta_{F}\right) Z$, where $Z$ is given by Eq. 22. The inert stage ends at a critical time $\tau_{c}$ at which the source term in Eq. 52 becomes important. In the vicinity of $\tau=\tau_{c}$, the evolution occurs over a short time in an ignition stage, during which a time variable like that of Appendix $B$ is of order unity. It is clear that the $\beta$ of Eq. 50 is the relevant expansion parameter in the description of the ignition stage by activationenergy asymptotics, and in this stage at leading order Eq. 52 becomes

$d \theta / d \tau=F$.

Appropriate stretched variables are basically those of Eq. B1, with $\sigma-\sigma_{c}$ replaced by $\tau-\tau_{c}$ provided that the $F_{c}$ of Eq. 51 is now defined to involve $d\left(\ln P_{s}\right) / d \tau$. The ignition analysis will then parallel that of Appendix B entirely. The only differences are that the inert solutions $\psi_{F}$ and $\psi_{O}$ are now more general functions than those of Eq. 49, and the matching condition $\phi_{-\infty}$ of Eq. B5 becomes instead $\beta\left[\theta_{I}\left(\eta, \tau_{c}\right)-1\right]$, where $\theta_{I}(\eta, \tau)$ is the more general inert solution indicated above.

If the rate of strain is large enough and varies slowly enough for the solutions in Eqs. 22 and 23 to become accurate before ignition occurs, then the results are like those in Eqs. B9 to B12, with $\zeta_{i} /\left(2 \sqrt{\sigma_{c}}\right)$ replaced by $\eta_{i} / \sqrt{2}$, and with the additional factor

$f=\delta^{2} /\left(\alpha_{O} t_{c}\right)$

appearing on the left-hand sides of Eqs. B10 and B12. The condition for the validity of Eqs. 22 and 23 at the time of ignition is that $f$ is small. Placing the factor $f<1$ in Eq. B12, for example, increases the value of $T_{O c}$ at ignition, thereby delaying ignition through the influence of the strain. Use of Eqs. 24 and 26 in Eq. 54 allows compression-produced autoignition times to be evaluated in the presence of timevarying strain under these conditions.

\section{CONCLUSIONS}

It may be concluded that, under many situations of interest in Diesels, ignition times may be estimated relatively straightforwardly from formulas like Eq. B12, provided that the rate parameters appropriate for a one-step, Arrhenius description of the ignition chemistry are available. These estimates may account not only for time-dependent mixing but also for influences of variable strain in the mixing layer. Strain delays ignition and in extreme cases may prevent it from occurring. The most favorable locations in mixing layers for ignition to occur therefore are positions of low rates of strain. The shortest compression-produced ignition times are those of Appendix B for strain-free conditions.

In the presence of strain, there are criticality conditions for ignition to occur, as shown in Appendix A. These conditions may be expressed in terms of a critical reduced Damköhler number of Eq. A22, having a value of about 0.5 . Effects of time-varying strain on ignition times may be calculated through the time-varying mixing-layer thickness $\delta$, given by Eqs. 24 and 26. Thus, many aspects of influences of strain and its variations on autoignition may be estimated.

The general formulation in Eqs. 2-13 encompasses a wide variety of ignition phenomena. Many of these phenomena have not been analyzed here. For example, situations in which variations of the strain rate $A$ through the mixing layer are important, as described by Eq. 3, have not been treated. Other outstanding problems have been identified in the preceding discussions. There are a number of problems in which pressure variations have only small influences on ignition, and these tend to necessitate consideration of partial differential equations during ignition stages. These problems have not been emphasized here; the focus has been on compressionproduced ignition. Strong compression simpli- 
fies the analysis by ultimately reducing the problems to ordinary differential equations in time. Thus, perhaps unexpectedly, many Diesel ignition problems are simpler than other ignition problems.

Research financed by the Commission of the European Communities within the frame of the JOULE Programme, by the National Swedish Board for Industrial and Technological Development (NUTEK) and by the Joint Research Committee for European automobile manufacturers (Fiat, Peugeot SA, Renault, Volkswagen and Volvo) within the IDEA Programme. We are indebted to N. Peters for suggesting these problems and to J. S. Kim for carrying out the numerical integrations for the figures.

\section{REFERENCES}

1. Kamimoto, T., and Kobayashi, H., Prog. Ener. Combust. Sci. 17:163-189 (1991).

2. Takagi, T., Fang, C.-Y., Kamimoto, T., and Okamoto, T., Combust. Sci. Technol. 75:1-12 (1991).

3. Liñán, A., and Williams, F. A., Fundamental Aspects of Combustion, Oxford University Press, New York, 1993.

4. Liñán, A., Acta Astronaut. 1:1007-1039 (1974).

5. Law, C. K., and Chung, S. H., Combust. Sci. Technol. 29:129-145 (1982).

6. Chung, S. H., and Law, C. K., Combust. Flame 52: 59-79 (1983).

7. Seshadri, K., and Treviño, C., Combust. Sci. Technol. 64:243-261 (1989).

8. Carrier, G. F., Fendell, F. E., and Marble, F. E., SIAM J. Appl. Math. 28:463-500 (1975).

9. Liñán, A., and Crespo, A., Combust. Sci. Technol. 14:95-117 (1976).

10. Niioka, T., Eighteenth Symposium (International) on Combustion, The Combustion Institute, Pittsburgh, 1981, pp. 1807-1813.

11. Liñán, A., INTA Report on AFOSR Grant 73-2535, March, 1975.

12. Williams, F. A., Combustion Theory, 2nd ed., Addison-Wesley, Menlo Park, CA, 1985, pp. 489-495.

13. Kassoy, D. R., and Liñán, A., Q.J. Mech. Appl. Math. 31:99-112 (1978).

Received 19 November 1992; revised 7 April 1993

\section{APPENDIX A: ANALYSIS OF IGNITION FOR CONSTANT RATE OF STRAIN}

The thermal-diffusive approximation is adopted, so that $A=A_{O}=A_{F}, \rho=\rho_{O}=\rho_{F}$ and $\lambda /\left(\rho c_{p}\right)=\alpha_{O}=\alpha_{F}$. The mixing-layer thickness becomes $\delta=\sqrt{\alpha_{O} / A_{O}}$, as indicated above Eq. 27. Relevant parameters are

$$
\begin{aligned}
& s=\nu_{O} Y_{F_{O}} / Y_{O o}, \quad \alpha=q /\left(c_{p} T_{O}\right), \\
& \beta=E /\left(R^{o} T_{O}\right),
\end{aligned}
$$

all three of which typically are large. For example, the air-to-fuel stoichiometric mass ratio for hydrocarbons is of order $s \approx 15$, the ratio $\alpha / s$ is approximately $\left(T_{a f}-T_{O}\right) / T_{O} \approx 6$, where $T_{a f}$ is the adiabatic flame temperature, and the nondimensional activation energy is perhaps $\beta \approx 20$ in ignition. In activationenergy asymptotics, the small parameter of expansion is $\beta^{-1}$.

The inert solution for the temperature field is

$T=T_{O}+\left(T_{F}-T_{O}\right) Z$,

where $Z$ is given by Eq. 22. The inert solutions for the concentration fields are similarly given by Eq. 23. The expansions

$$
\begin{aligned}
T & =T_{O}\left(1-c Z+\beta^{-1} \varphi+\cdots\right), \\
Y_{F} & =Y_{F o}\left(Z_{F}+\alpha^{-1} \beta^{-1} y_{F}+\cdots\right), \\
Y_{O} & =Y_{O o}\left(1-Z_{O}+s \alpha^{-1} \beta^{-1} y_{O}+\cdots\right)
\end{aligned}
$$

are sought, where the nonnegative nondimensional temperature difference is

$c=\left(T_{O}-T_{F}\right) / T_{O}$.

Introduction of Eq. 16 and of these nondimensionalizations and expansions into Eqs. 29-32 leads to the system of equations

$$
\begin{aligned}
\eta \frac{d y_{F}}{d \eta}+\frac{1}{L_{F}} \frac{d^{2} y_{F}}{d \eta^{2}} & =\eta \frac{d y_{O}}{d \eta}+\frac{1}{L_{O}} \frac{d^{2} y_{O}}{d \eta^{2}} \\
= & -\eta \frac{d \varphi}{d \eta}-\frac{d^{2} \varphi}{d \eta^{2}} \\
= & D\left[Z_{F}+y_{F} /(\alpha \beta)\right]^{n} \\
& \times\left(1-Z_{O}\right)^{m} e^{\varphi-\beta c z}
\end{aligned}
$$

subject to the boundary conditions that $\varphi, y_{F}$, and $y_{O}$ approach zero as $\eta \rightarrow \pm \infty$. Here the 
relevant Damköhler number is

$D=\alpha \beta A_{O}^{-1} B Y_{F o}^{n-1} Y_{O o}^{m} e^{-E /\left(R^{o} T_{o}\right)}$.

In the reaction-rate term in Eq. A5, expansions have been introduced in which terms that are not of leading order in any ignition regime have been dropped.

When $\beta c$ is of order unity or smaller, reaction occurs everywhere in the mixing layer, and unless $\alpha$ is unreasonably small $y_{F} /(\alpha \beta)$, which describes the effect of fuel depletion, is of higher order in the reaction term in Eq. A5 and can be neglected in the first approximation. In this case, Eqs. 22 and 23 enable the equation for $\varphi$ in Eq. A5 to be solved to yield $\varphi(\eta)$ for given values of $\beta c, m, n, L_{F}, L_{O}$, and $D$; the functions $y_{F}(\eta)$ and $y_{O}(\eta)$ can be calculated later. The solution for $\varphi(\eta)$ will exist only for $D<D_{I}$, where $D_{I}$ is the ignition Damköhler number, of order unity. This twovalued solution can be determined numerically for different values of $\beta c, m, n, L_{F}$, and $L_{O}$, and $D_{I}$ can similarly be calculated as a function of these parameters. Parametric results for $m=n=L_{F}=L_{O}=1$ are available [4].

When $\beta c$ is large, the reaction rate is negligibly small except where $\beta c Z$ is of order unity. It is therefore useful to introduce the variable

$\xi=\beta c Z$.

For large $\xi$, away from the oxidizer side of the mixing layer, the chemistry is frozen, and the solutions are

$$
\begin{aligned}
\varphi & =\varphi_{m}(1-Z), \quad y_{F}=y_{F_{m}}\left(1-Z_{F}\right), \\
y_{O} & =y_{O m}\left(1-Z_{O}\right),
\end{aligned}
$$

where the peak values, $\varphi_{m}, y_{F m}$, and $y_{O m}$, are to be determined by matching with the solution in the reaction region, where $\xi$ is of order unity.

In the reaction zone $Z$ is small and therefore can be approximated from Eq. 22 as

$Z=e^{-\eta^{2} / 2}(\eta \sqrt{2 \pi})^{-1}$.

The value of $\eta$ in the reaction zone, denoted by $\eta_{r}$, may be estimated from Eqs. A7 and A9 by putting $\xi=1$, giving

$e^{-\eta_{r}^{2} / 2}\left(\eta_{r} \sqrt{2 \pi}\right)^{-1}=(\beta c)^{-1}$.

The values of $Z_{F}$ and $Z_{O}$ also are small there, so that $1-Z_{O} \approx 1$ in Eq. A5, and the expansions

$$
\begin{aligned}
Z & \approx \frac{e^{-\eta_{r}^{2} / 2}}{\sqrt{2 \pi} \eta_{r}} e^{-\eta_{r}\left(\eta-\eta_{r}\right)}, \\
Z_{F} & \approx \frac{e^{-\eta_{r}^{2} L_{F} / 2}}{\sqrt{2 \pi L_{F}} \eta_{r}} e^{-\eta_{r}\left(\eta-\eta_{r}\right) L_{F}},
\end{aligned}
$$

or, from Eqs. A7 and A10, equivalently,

$$
\xi=e^{-\eta_{r}\left(\eta-\eta_{r}\right)}
$$

and

$Z_{F}=Z_{F r} \xi^{L_{F}}$

may be employed, where

$Z_{F r}=(\beta c)^{-L_{F}}\left(\sqrt{2 \pi} \eta_{r}\right)^{L_{F}-1} L_{F}^{-1 / 2}$.

These exponential profiles, in $\eta$, of $\xi$ and $Z_{F}$, given by Eqs. $\mathrm{A} 12$ and $\mathrm{A} 13$, result from approximating in the reaction zone the convective velocity $\eta$ appearing in the first-derivative terms in Eq. A5 by a constant value $\eta_{r}$; they are justified by the moderately large value of $\eta_{r}$. The same approximation can be used when writing the conservation equation for $\varphi$ in the reaction zone, so that Eq. A5 with fuel depletion neglected becomes

$\eta_{r} \frac{d \varphi}{d \eta}+\frac{d^{2} \varphi}{d \eta^{2}}+D Z_{F r}^{n} \xi^{n L_{F}} e^{\varphi-\xi}=0$,

to be solved with the boundary conditions

$$
\begin{aligned}
& \varphi \rightarrow \varphi_{m} \text { as } \eta-\eta_{r} \rightarrow-\infty, \\
& \varphi \rightarrow 0 \text { as } \eta-\eta_{r} \rightarrow \infty,
\end{aligned}
$$

the former obtained from matching to Eq. A8. Here $\varphi_{m}$ is an eigenvalue to be determined as part of the solution as a function of $D$.

In this same approximation, Eq. A5 gives for the fuel concentration

$$
\eta_{r} \frac{d y_{F}}{d \eta}+\frac{1}{L_{F}} \frac{d^{2} y_{F}}{d \eta^{2}}-D Z_{F r}^{n} \xi^{n L_{F}} e^{\varphi-\xi}=0,
$$


with the boundary conditions

$y_{F} \rightarrow y_{F_{m}}$ as $\eta-\eta_{r} \rightarrow-\infty$,

$y_{F} \rightarrow 0$ as $\eta-\eta_{r} \rightarrow \infty$,

where $y_{F m}$ must be determined as part of the solution. From Eqs. A15 to A18 we obtain the equations

$$
\begin{gathered}
\eta_{r}\left(\varphi+y_{F}\right)+\frac{d}{d \eta}\left(\varphi+y_{F} / L_{F}\right) \\
=\eta_{r}\left(\varphi_{m}+y_{F m}\right)=0
\end{gathered}
$$

so that $y_{F m}=-\varphi_{m}$. Thus, with $\varphi_{m}$ of order unity, the fuel consumption in the reaction term in Eq. A5 can be neglected when describing the ignition process, so long as $(\alpha \beta)^{-1} \ll$ $Z_{F r}$, that is, from Eq. A14,

$$
\alpha \beta(\beta c)^{-L_{F}}\left(\eta_{r} \sqrt{2 \pi}\right)^{L_{F}-1} L_{F}^{-1 / 2} \gg 1 .
$$

Usually $\alpha$ is large enough to insure that Eq. A20 is satisfied and that therefore fuel depletion can be neglected.

When solving Equation A15 it is useful to use $\xi$ defined by Eq. A7 as the independent variable; Eq. A15 then becomes

$$
\frac{d^{2} \varphi}{d \xi^{2}}+\Delta \xi^{n L_{F}-2} e^{\varphi-\xi}=0,
$$

where $\Delta=\eta_{r}^{-2} D Z_{F r}^{n}$, or

$$
\begin{aligned}
\Delta= & \eta_{r}^{-2}\left\{\left(\eta_{r} \sqrt{2 \pi}\right)^{L_{F}-1}(\beta c)^{-L_{F}}\right\}^{n} \\
& \times \alpha \beta B A_{O}^{-1} Y_{F o}^{n-1} Y_{O o}^{m} e^{-E / R^{\circ} T_{O}},
\end{aligned}
$$

is the reduced Damköhler number, of order unity, under near-ignition conditions for $\beta c \gg$ 1. It may be recalled that $\eta_{r}$ in Eq. A22 is determined by Eq. A10. Equation A21 must be solved with the boundary conditions

$\varphi=0$ at $\xi=0, \quad \varphi \rightarrow \varphi_{m}$ as $\xi \rightarrow \infty$,

where the increment in temperature $\varphi_{m}$ must be calculated as a function of $\Delta$ for different values of the parameter $n L_{F}$. Because we can expect $\varphi_{m}$ to be a two-valued function of $\Delta$ for $\Delta$ smaller than an ignition value $\Delta_{l}$, and no solution to exist for values of $\Delta>\Delta_{l}$, it is more convenient to pose the problem as that of finding the value of the Damköhler num- ber $\Delta$ that results in a given value of the temperature increment $\varphi_{m}$. The function $\Delta=$ $\Delta\left(\varphi_{m}, n L_{F}\right)$ will exhibit a maximum $\Delta_{I}=\Delta_{I}$ $\left(n L_{F}\right)$ that characterizes the ignition conditions.

In the particular case $n L_{F}=2$, the solution can be obtained in closed form by using $\Psi=$ $\varphi-\xi$ as the independent variable. A first integral of Eq. A21 in this case is

$\Psi_{\xi}^{2}-\Psi_{\xi}^{2}(0)=2 \Delta\left(1-e^{\Psi}\right)$

where $\Psi_{\xi}^{2}(0)=1-2 \Delta$. Thus $\Delta \leq 1 / 2$; the ignition value of $\Delta$ for $n L_{F}=2$ is $\Delta_{I}=1 / 2$. The stable, lower branch of the curve $\varphi(\xi, \Delta)$ is then given by

$$
\xi=\int_{\Psi}^{0}\left\{1-2 \Delta e^{\Psi}\right\}^{-1 / 2} d \Psi
$$

or

$$
\varphi=\Psi+\xi=2 \ln \left(\frac{1+\sqrt{1-2 \Delta e^{\Psi}}}{1+\sqrt{1-2 \Delta}}\right)
$$

and

$\varphi_{m}=2 \ln \{2 /(1+\sqrt{1-2 \Delta})\}$.

Fortuitously, the value $n L_{F}=2$ often may be reasonably accurate for many hydrocarbon fuels in air.

\section{APPENDIX B: ANALYSIS OF IGNITION BY COMPRESSION IN THE UNSTRAINED MIXING LAYER}

Let

$\phi=\beta(\theta-1), \quad v=F_{c} \beta\left(\sigma-\sigma_{c}\right)$,

where $\beta$ and $F_{c}$ are defined in Eqs. 50 and 51. Substitution into Eqs. 45 and 47 and expansion to leading order for the ignition stage then gives

$d \phi / d v=\Lambda \psi_{F}^{n} \psi_{O}^{m} e^{\phi+v}$,

where

$\Lambda=\frac{q B_{c} Y_{F o}^{n} Y_{O o}^{m} t_{c} e^{-E /\left(R^{\circ} T_{O c}\right)}}{F_{c} c_{p} T_{O 0}\left(P_{s c} / P_{s 0}\right)^{(\gamma-1) / \gamma}}$. 
The manner in which $\phi+v$ arises in the exponent is through the expansion

$$
\begin{aligned}
\frac{E}{R^{o} T_{O 0}} & \left(\frac{P_{s c}}{P_{s 0}}\right)^{-(\gamma-1) / \gamma}\left[1-\frac{1}{\theta}\left(\frac{P_{s c}}{P_{s}}\right)^{(\gamma-1) / \gamma}\right] \\
= & \frac{E}{R^{o} T_{O c}}\left\{1-\left(1-\frac{\phi}{\beta}+\cdots\right)\right. \\
& \left.\times\left[1-F_{c}\left(\sigma-\sigma_{c}\right)+\cdots\right]\right\}
\end{aligned}
$$

which shows that the $v$ term is the effect of the $T_{O}$ increase caused by compression. The matching condition for Eq. B2 is found from Eq. 48 to be

$$
\begin{aligned}
\phi & \rightarrow-\beta\left(1-\theta_{F}\right) \frac{1}{2} \operatorname{erfc}\left(\frac{\zeta / 2}{\sqrt{\sigma_{c}}}\right) \\
& \equiv \phi_{-\infty} \text { as } v \rightarrow-\infty .
\end{aligned}
$$

If $\psi_{F}$ and $\psi_{O}$ are independent of $\phi$ and $v$, (for example, given by Eq. 49), then the integral of Eq. B2 is

$e^{-\phi_{-\infty}}-e^{-\phi}=\Lambda \psi_{F}^{n} \psi_{O}^{m} e^{v}$

which has $\phi \rightarrow \infty$ (thermal runaway) when

$v=-\phi_{-\infty}-\ln \left(\Lambda \psi_{F}^{n} \psi_{O}^{m}\right)$,

the minimum value of which (over all values of $\zeta$ ) is selected to be zero so that, by definition, the thermal runaway first occurs at $\sigma=\sigma_{c}$. If the subscript $i$ is employed to denote the value of $\zeta$ at which the minimum occurs, then from Eqs. 49, B5, and B7 it is found that

$$
\begin{aligned}
\exp \left\{\beta\left(1-\theta_{F}\right) \frac{1}{2} \operatorname{erfc}\left(\frac{\zeta_{i} / 2}{\sqrt{\sigma_{c}}}\right)\right\} \\
=\Lambda\left[\frac{1}{2} \operatorname{erfc}\left(\frac{\zeta_{i}}{2} \sqrt{\frac{L_{F}}{\sigma_{c}}}\right)\right]^{n} \\
\times\left[1-\frac{1}{2} \operatorname{erfc}\left(\frac{\zeta_{i}}{2} \sqrt{\frac{L_{O}}{\sigma_{c}}}\right)\right]^{m}
\end{aligned}
$$

and

$$
\begin{aligned}
n \sqrt{L_{F}} & {\left[\frac{1}{2} \operatorname{erfc}\left(\frac{\zeta_{i}}{2} \sqrt{\frac{L_{F}}{\sigma_{c}}}\right)\right]^{-1} } \\
& -m \sqrt{L_{O}}\left[1-\frac{1}{2} \operatorname{erfc}\left(\frac{\zeta_{i}}{2} \sqrt{\frac{L_{O}}{\sigma_{c}}}\right)\right]^{-1} \\
= & \beta\left(1-\theta_{F}\right),
\end{aligned}
$$

the last of which is obtained by differentiation $\left(d v / d \zeta=0\right.$ at the location $\zeta=\zeta_{i}$ of the minimum) and serves to determine the value of $\zeta_{i}$. With $t_{c}$ not yet defined precisely, it is possible to select $\sigma_{c}=1$ and then determine $t_{c}$ by substituting Eqs. 51 and B3 into Eq. B8. The result is

$$
\begin{gathered}
\frac{\gamma q B_{c} Y_{F o}^{n} Y_{O o}^{m} e^{-E /\left(R^{o} T_{O c}\right)}}{(\gamma-1) c_{p} T_{O c}\left[d\left(\ln P_{s}\right) / d t\right]_{t=t_{c}}} \\
\times\left[\frac{1}{2} \operatorname{erfc}\left(\frac{\zeta_{i}}{2} \sqrt{L_{F}}\right)\right]^{n} \\
\times\left[1-\frac{1}{2} \operatorname{erfc}\left(\frac{\zeta_{i}}{2} \sqrt{L_{O}}\right)\right]^{m} \\
=\exp \left\{\frac{E\left(T_{O c}-T_{F c}\right)}{R^{o} T_{O c}^{2}}\left[\frac{1}{2} \operatorname{erfc}\left(\frac{\zeta_{i}}{2}\right)\right]\right\},
\end{gathered}
$$

which with $\zeta_{i}$ determined by Eq. B9 provides $t_{c}$ mainly through the variation of $e^{-E /\left(R^{o} T_{O c}\right)}$, in which $T_{O c}=T_{O 0}\left(P_{s c} / P_{s 0}\right)^{(\gamma-1) / \gamma}$ according to Eq. 50.

For large values of $\beta\left(1-\theta_{F}\right)$, Eq. B9 gives

$$
\begin{aligned}
\zeta_{i}= & \left(2 / \sqrt{L_{F}}\right) \\
& \times \operatorname{erfc}^{-1}\left\{\left(2 n \sqrt{L_{F}}\right) /\left[\beta\left(1-\theta_{F}\right)\right]\right\},
\end{aligned}
$$

where $\operatorname{erfc}^{-1}$ denotes the inverse of the complementary error function, and manipulations like those of Eqs. A9 and A10, also using 
$\beta\left(1-\theta_{F}\right)=E\left(T_{O 0}-T_{F 0}\right) /\left(R^{o} T_{O 0} T_{O c}\right)$, reduce Eq. B10 to

$$
\begin{aligned}
& \frac{\gamma q B_{c} Y_{F o}^{n} Y_{O o}^{m} e^{-E /\left(R^{o} T_{O c}\right)}}{(\gamma-1) c_{p} T_{O c}\left[d\left(\ln P_{s}\right) / d t\right]_{t=t_{c}}} \\
& \times\left[\frac{n \sqrt{L_{F}} R^{o} T_{O 0} T_{O c}}{E\left(T_{O 0}-T_{F 0}\right)}\right]^{n} \\
&=\exp \left\{\left(n L_{F}\right)^{1 / L_{F}}\right. \\
&\left.\times\left[\frac{E\left(T_{O 0}-T_{F 0}\right)}{\sqrt{\pi} \zeta_{i} R^{o} T_{O 0} T_{O c}}\right]^{\left(L_{F}-1\right) / L_{F}}\right\},
\end{aligned}
$$

which is more direct than Eqs. B9 and B10 to employ for determining when ignition occurs.

If $\beta\left(1-\theta_{F}\right)$ becomes too large, then in the reaction region about $\zeta=\zeta_{i}$, identified above, the inert fuel concentration is so small that fuel consumption becomes significant during the ignition stage. It then becomes necessary to determine $\psi_{F}$ from Eq. 45 during the ignition stage, matching to Eq. 49 as $v \rightarrow-\infty$. In the ignition stage at the reaction region, $\psi_{O}=1$ to leading order, and

$\chi \equiv \beta \psi_{F} q Y_{F o} /\left(c_{p} T_{O c}\right)$

is of order unity, so that it becomes appropriate to redefine $\Lambda$ to include the additional factor $\left[\left(c_{p} T_{O c}\right) /\left(q Y_{F o} \beta\right)\right]^{n}$, obtaining from Eqs. 45 and 47

$$
-d \chi / d v=d \phi / d v=\Lambda \chi^{n} e^{\phi+v},
$$

since diffusive effects are of higher order. Integration of the first of these equalities, subject to the matching conditions, gives

$$
\begin{aligned}
\chi= & \frac{\beta q Y_{F o}}{\sqrt{\pi L_{F}} \zeta c_{p} T_{O c}} e^{-\left(\zeta^{2} / 4\right) L_{F}} \\
& -\frac{\beta\left(1-\theta_{F}\right)}{\sqrt{\pi} \zeta} e^{-\zeta^{2} / 4}-\phi
\end{aligned}
$$

under the present conditions, where the selection $\sigma_{c}=1$ has again been made. The value of $\zeta$ in the reaction region is suitably large for the terms in Eq. B15 to be of order unity. A single ordinary differential equation (with $\zeta$ as a parameter) is obtained from the second equality in Eq. B14 when Eq. B15 is employed in the reaction term. The fuel depletion enters through the $\phi$ term in Eq. B15, which reduces $x$ as $\phi$ increases. The character of the solution to the resulting differential equation has been fully explored [11]. The reactant depletion can prevent thermal runaway and give rise to premixed-flame propagation away from an ignition point. 